\title{
r-process nucleosynthesis in a double neutron-star merger: the case of GW170817
}

\author{
Giulia Stratta ${ }^{* \dagger}$ \\ INAF/OAS-Bologna \\ INFN-Firenze E-mail: giulia.stratta@inaf.it
}

\begin{abstract}
Double neutron star (NS-NS) mergers have been predicted as powerful gravitational waves and electromagnetic sources and are among the main targets of current gravitational wave detectors. These sources are also expected to be important astrophysical sites for rapid neutron capture (rprocess) nucleosynthesis at the origin of the "Kilonova" (or "macronova") emission. So far only one NS-NS sytem (GW 170817) has been discovered with the current ground based gravitational wave laser interferometers. In this proceeding I will review the main observational properties and the still ongoing interpretation of the GW 170817 optical/NIR counterpart, AT2017gfo, recognized as the first convincing evidence of the existence of kilonovae and of the r-process nucleosynthesis from NS-NS system.
\end{abstract}

Frontier Research in Astrophysics - III (FRAPWS2018)

28 May - 2 June 2018

Mondello (Palermo), Italy

* Speaker.

${ }^{\dagger}$ A footnote may follow. 


\section{Introduction}

Gravitational waves (GWs) have been detected for the first time with the ground based laser interferometer GW observatories Advanced LIGO (aLIGO [1]) and Advanced Virgo [2] nearly 100 years from their theoretical prediction. aLIGO and Advanced Virgo are most sensitive to the high frequency range of the GW spectrum (about 10-1000 Hz). At these frequencies we expect to see a number of astrophysical source classes, most of which of transient nature and known to emit also in the electromagnetic spectrum. In particular, compact binary coalescences ( $\mathrm{CBCs})$ as the merger of two neutron stars (NS) or a neutron star and a stellar-mass black hole (BH) have the best configuration to be powerful sources of high frequency GWs and at the same time are thought to be the progenitor of the well known short Gamma Ray Bursts (GRBs) and their associated afterglows as well as of the theoretically predicted emission called "kilonova" (or "macronova"). The latter, contrary to short GRBs, lacked of compelling evidence for a long time since its prediction in the late seventies. Core collapsing supernovae as well as instability phenomena on the surface of neutron stars, likely at the origin of Soft Gamma Repeaters and Anomalous X-ray Pulsars, are also expected to emit high frequency GWs, however with larger uncertainties with respect to the CBC class both on the expected waveform and energy output.

After two years of aLIGO and Advanced Virgo observational campaigns, the first gravitational wave (GW) transient catalog of compact binary coalescences (GWTC-1) counts $10 \mathrm{BH}-\mathrm{BH}$ and 1 NS-NS GW-detected systems [3]. Only in the latter case (the NS-NS system, named GW170817) the electromagnetic (EM) counterpart has been discovered, greatly enhancing the scientific output that would had been obtained from a gravitational wave only detection. GW170817 has became a milestone in several aspects that goes from fundamental physics (e.g. [4],[5]) to astrophysics (e.g. [6]) and cosmology (e.g. [7]). In this proceeding I will describe the observations and still ongoing interpretations of the early ( $<1$ month from the merger) optical/NIR counterpart of GW170817 that has been identified with the first convincing evidence of a "kilonova", the predicted emission from NS-NS merger systems, the properties of which encode crucial implications on the most heavy element enrichment of our Universe.

\section{Expected electromagnetic emission from NS-NS merger systems}

During the last two decades mounting empirical evidence have supported the hypothesis that short GRBs originate from the coalescence of two NSs or a NS-BH systems, among which: 1) the lack of any supernova associated with a short GRB (contrary to long GRBs); 2) the off-set from their host galaxy center, interpreted as a hint of an old population that could in principle reach large distances from their formation region; 3) the mixed nature of short GRB host galaxies, both of late and early types, indicating a large range of lifetime of the progenitors, in line with the expected timescale distribution of $\mathrm{CBCs}$ from the epoch of their formation to the merging phase (see e.g. [8] for a review).

Another electromagnetic counterpart of NS-NS or NS-BH merging systems theoretically predicted since the late seventies [9] is the so called "kilonova" (or "macronova"), an emission powered by the radioactive decay of heavy isotopes synthesized through rapid neutron capture ( $\mathrm{r}$ processes) in the neutron-rich matter ejected during the merger. Contrary to the well known short 
GRBs phenomenology, kilonova emission lacked of compelling empirical evidence for a long time. One of the most accredited kilonova identification before GW170817 was found in the afterglow of the short GRB 130603B [10]. From an observation taken $\sim 7$ days after GRB 130603B, an unexpected significant flux increase (nearly 5 magnitudes) from the afterglow extrapolation was inferred and interpreted as a rising extra-component. The luminosity, color and timescale of this possible component were consistent with the predictions from kilonova models (see [10] and reference therein). Other, possible cases of kilonova signatures were found in the afterglows of the short GRB 050709 and the peculiar long GRB 060614 [11], the short GRB 080503 ([12]; [13]), 160821B ([14]; [15]) and 150101B [16].

\section{AT2017gfo: observations}

On August the 17th, 2017, at $T_{0}=12: 41: 04$ UT a gravitational wave signal with $\mathrm{S} / \mathrm{N}=32.4$ was detected with the LIGO-Virgo network and found to be consistent with a NS-NS merger at $40 \mathrm{Mpc}$ (GW170817, [4]). At the same time, a short GRB (GRB170817) triggered the gamma-ray detectors onboard the INTEGRAL and Fermi satellites ([17], [18]), with a delay of $1.7 \mathrm{~s}$ with respect to the NS-NS merger epoch (see [6] and reference therein). The sky position of the GW source computed using the laser interferometer network data was located in the Southern Hemisphere with a centroid at R.A. $=12^{h} 57^{m}$ and Dec. $=-17^{\circ} 51^{\prime}$ and an uncertainty of $31 \mathrm{deg}^{2}$ at $90 \%$ probability, fully contained within the much larger error box computed from the Fermi/GBM data analysis of the GRB (1100 deg 2 at $90 \%$ confidence, [17]).

A massive follow-up observational campaign of GW170817/GRB170817 started immediately after the first communication at $T_{0}+27 \mathrm{~min}$ (see [6] and reference therein). At $T_{0}+11$ hours a bright optical source not present in the archival images was discovered within the lenticular galaxy NGC4993 at $40 \mathrm{Mpc}$ ([19], [6] ). The source, officially (IAU) named AT2017gfo ${ }^{1}$ and located at right ascension $13^{h} 09^{m} 48^{s} .085 \pm 0.018$ and declination $-23^{o} 22^{\prime} 53^{\prime \prime} .343 \pm 0.218$ (J2000 equinox, [19]) showed a rapid transient nature with strong color evolution and it was subsequently identified as the electromagnetic counterpart of GW 170817. In about 11 days AT2017gfo decreased in intensity of 6 magnitudes in the $\mathrm{i}$ band (from 17 to $23 \mathrm{mag}$ ) and of $5 \mathrm{mag}$ in 5 days in the $\mathrm{g}$ band (see e.g. [20]).

Spectroscopic observations of exceptional quality of AT2017gfo have been taken from T0+1.5 days to T0+17.5 days with the VLT/X-shooter and FORS2, Gemini-South/GMO, NTT/EFOSC2 and SOFI $[20,21]$. Additional spectra have been taken from $\mathrm{T} 0+1.5$ and $\mathrm{T} 0+9.5$ days with the SOAR and Magellan telescopes [22] and with the Hubble Space Telescope at T0+5.5 days in UV [22] and between T0+4.9 and T0+10.6 in the NIR [23]. During the first night, AT2017gfo spectrum showed a blue, bright continuum with a peak at $6000 \AA$ and luminosity of $3.2 \times 10^{41} \mathrm{erg} \mathrm{s}^{-1}$. The overall shape was well modelled by black body with $\mathrm{T} \sim 5500 \mathrm{~K}$ and a spherical equivalent radius of $\sim 8 \times 10^{14} \mathrm{~cm}$, that after 1.5 days from the merger implies an ejecta expansion velocity of 0.2c [20]. Evidence of broad absorption features between 7000 and $9000 \AA$ have been tentatively identified with CsI and TeI lines blueshifted by the expansion velocity of the ejecta [21]. The spectra taken few days later showed a peak emission at longer wavelengths $(11000 \AA$ at $\mathrm{T} 0+5.5$

\footnotetext{
${ }^{1}$ other names of the same source were SSS17a and DLT17ck
} 
days) consistently with a rapid cooling of the ejecta, indeed too rapid to be compatible with a supernova-like source. Additional absorption features became visible but could not be identified (e.g. [20], [22], [23]).

\section{A kilonova associated with a binary neutron star merger}

The short GRB 170817 and its following afterglow as well as the early optical/NIR counterpart AT2017gfo associated with GW170817 astonishingly confirmed the main electromagnetic emitting components theoretically predicted from NS-NS systems (see Section 1). GW170817 represent the first direct association of a short GRB with a NS-NS merger progenitor and the main temporal and spectral properties of AT2017gfo are consistent with the theoretical expectations from a kilonova emission, representing the first compelling evidence on the existence of these important astrophysical sources.

The general picture of kilonova modelling predicts that during the merger of two NSs, a fraction of neutron rich matter $\left(\sim 10^{-2}-10^{-3} \mathrm{M}_{\odot}\right)$ is stripped from the system and ejected into space with an almost isotropic geometry. The temperature and neutron density of the ejected material are such that rapid neutron capture (r-process) synthesis of heavy and instable isotopes can originate, making NS-NS mergers optimal candidates for r-process elements cosmological sites together with supernovae (e.g. [9]). Radioactivity from the freshly synthesized unstable elements heats the ejecta that ultimately radiates the thermal emission (e.g. [24], [25] and reference therein).

The basic properties of kilonova emission as the peak luminosity, peak time scale and temperature depend on few main parameters among which the ejecta mass, expansion velocity and matter opacity $(k)$. While the ejecta mass and velocity can be fairly well estimated via numerical simulations, the matter opacity is still affected by large uncertainties that strongly depend on the poorly defined production yield of the heaviest atomic species, in particular lanthanides and actinides with atomic mass number $A=58-71$ and $A=90-103$, respectively. "Lanthanides-rich" ejecta are expected to produce large opacities $\left(k \gtrsim 10 \mathrm{~cm}^{2} \mathrm{gr}^{-1}\right)$ and ultimately a kilonova emission peaking in the NIR spectral regime with longer timescales and lower intensities than the more blue emission expected from "lanthanides-poor" ejecta (see e.g. [26] and reference therein).

In the case of AT2017gfo, the observations were consistent with an ejecta of $\sim 0.05 \mathrm{M}_{\odot}$ and velocity $v / c \sim 0.1-0.3$ (e.g. [27], [28], [20], [29]). The marked blue to red evolution of AT2017gfo has been interpreted as the evidence of inhomogeneous properties of the ejecta, where lanthanides-poor regions are responsible of the blue, early emission while lanthanides-rich regions dominate at late times (e.g. [30], [28]). Neutrino irradiation from a central hpermassive or supramssive NS remnant and/or an accretion disk have been invoked as possible mechanisms that would halt r-process synthesis (thus lanthanides formation) prevalently at high latitudes (e.g. [29]). A full understanding of the properties of the ejecta and the ejection mechanisms is still incomplete and strong efforts in semi-analytic modellings and fully numerical simulations are still ongoing (e.g. [31]). Nevertheless, the observations and the so far interpretation clearly indicate that NS-NS mergers can be considered as powerful cosmological sites not only of the most heavy (i.e. beyond the second r-process peak at $\mathrm{A}=130$ ), as originally predicted from the very first kilonova formulation, but also lighter r-process elements. This fact, together with the inferred NS-NS merger cosmic 
rate of $1540_{-1220}^{+3200} \mathrm{Gpc}^{-3} \mathrm{yr}^{-1}$ ([5]) makes NS-NS mergers optical candidates to be the dominant cosmological sites of r-process nucleosynthesis in our Universe (e.g. [32]).

According to NS-NS merger theoretical models and to GW170817/GRB170817/AT2017gfo observations, in principle all short GRBs should be associated with a kilonova emission. The fact that only in few cases a kilonova component has been found as an extra component in the opticalNIR afterglow (see Section 1) is likely due to the typical brighter afterglow luminosity. In order to investigate on the possible peak luminosity range of kilonovae, comparison of optical-NIR afterglows of short GRBs and kilonova AT2017gfo luminosity light curves have been performed (e.g. [33],[34]). Robust evidence that not all sGRBs are consistent with being associated with a kilonova emission as bright as AT2017gfo has been found, with possible kilonova luminosities up to 5 times fainter than AT2017gfo. These results are posing interesting constraints on emission mechanism/existence of a kilonova and their implications are still under study (Rossi, Stratta, Maiorano et al. 2019, submitted to MNRAS).

\section{Summary and Future perspectives}

Kilonovae have been predicted as powerful factories of the most heavy r-process elements in our Universe since late seventies and predicted to emit in the optical/NIR bands (e.g. [9], [25]). Despite their importance, kilonova lacked of compelling empirical evidence for a long time due to their challenging identification in optical surveys and/or as additional component in short GRB optical afterglows. Among the main reasons there are the fast evolution (with respect to supernovae) and low luminosities (with respect to GRB afterglows) of their emission.

The combination of gravitational waves and electromagnetic counterpart detection from the same source (GW170817/AT2017gfo) has enabled for the first time to confirm the association of kilonovae with the merger of two NSs, definitively assessing their existence and ultimately their relevance in cosmic r-process element production. Exceptional amount of data and high quality spectra of the GW170817 optical counterpart, AT2017gfo, with long temporal coverage have enable to gain crucial insights on the physics of neutron stars and of their merging phase. Among the main results from AT2017gfo: 1) NS-NS merger ejecta shows evidence of inhomogeneous chemical composition with both light and heavy r-process elements, in agreement with the most recent kilonova modellings (e.g. [30]); 2) compelling element identifications from absorption spectral features was prevented by the poorly known properties of the synthesized atomic species and the likely blendening of absorption lines; 3 ) estimated total mass of neutron-rich matter ejected into space, about $0.05 \mathrm{M}_{\odot}$, is consistent with NS-NS being dominant sources of r-process elements (e.g. [32]); 4) comparison of AT2017gfo with past short GRB afterglows revealed that kilonova emission can be much fainter up to 5 times or more than AT2017gfo. Implications of this result are still under evaluation (Rossi, Stratta, Maiorano et al. 2019, submitted to MNRAS).

The next observing run of LIGO and Virgo (O3) is planned to start on April 2019 and to last one year. Expected detection rates of NS-NS during O3 is still highly uncertain and goes from 1 per month up to 1 per year, while NS-BH systems are still consistent with null detection in one year. By the end of 2024, the interferometer network is expected to work at full sensitivity with at least 4 detectors: by that time a detection rate of 11-180/yr NS-NS is expected [35]. Next generation of laser interferometers as the Einstein Telescope (e.g. [36]) will increase the current 
second generation sensitivity of one order of magnitude and will be able to detect GWs from NS-NS and NS-BH up to high redshift with an expected rate of several hundreds per year. An AT2017gfo-like kilonova would be detectable up to redshift 0.5 in the optical and 0.2 in the NIR by ground-based very large telescopes. With the JWST and the future ELT, it will be possible to detect AT2017gfo-like emission at redshift larger than one (see e.g. Rossi, Stratta, Maiorano et al. 2019, submitted to MNRAS), making the next decades a nascent golden era for multi-messenger astronomy in general and for kilonova characterizations in particular, providing crucial insights on the r-process nucleosynthesis in our Universe.

\section{References}

[1] T. L. S. Collaboration, J. Aasi, B. P. Abbott, R. Abbott, T. Abbott, M. R. Abernathy et al., Advanced LIGO, Classical and Quantum Gravity 32 (2015) 074001.

[2] F. Acernese, M. Agathos, K. Agatsuma, D. Aisa, N. Allemandou, A. Allocca et al., Advanced Virgo: a second-generation interferometric gravitational wave detector, Classical and Quantum Gravity 32 (2015) 024001.

[3] T. L. S. Collaboration, the Virgo Collaboration, B. P. Abbott, R. Abbott, T. D. Abbott, S. Abraham et al., GWTC-1: A Gravitational-Wave Transient Catalog of Compact Binary Mergers Observed by LIGO and Virgo during the First and Second Observing Runs, arXiv:1811.12907 [astro-ph, physics:gr-qc] (2018) .

[4] B. P. Abbott, R. Abbott, T. D. Abbott, F. Acernese, K. Ackley, C. Adams et al., Gravitational Waves and Gamma-Rays from a Binary Neutron Star Merger: GW170817 and GRB 170817a, The Astrophysical Journal 848 (2017) L13.

[5] LIGO Scientific Collaboration and Virgo Collaboration, B. Abbott, R. Abbott, T. Abbott, F. Acernese, K. Ackley et al., GW170817: Observation of Gravitational Waves from a Binary Neutron Star Inspiral, Physical Review Letters 119 (2017) 161101.

[6] B. P. Abbott, R. Abbott, T. D. Abbott, F. Acernese, K. Ackley, C. Adams et al., Multi-messenger Observations of a Binary Neutron Star Merger*, The Astrophysical Journal Letters 848 (2017) L12.

[7] The LIGO Scientific Collaboration and The Virgo Collaboration, The 1M2H Collaboration, The Dark Energy Camera GW-EM Collaboration and the DES Collaboration, The DLT40 Collaboration, The Las Cumbres Observatory Collaboration, The VINROUGE Collaboration et al., A gravitational-wave standard siren measurement of the Hubble constant, Nature 551 (2017) 85.

[8] E. Berger, Short-Duration Gamma-Ray Bursts, Annual Review of Astronomy and Astrophysics 52 (2014) 43.

[9] J. M. Lattimer and D. N. Schramm, BLACK-HOLE-NEUTRON-STAR COLLISIONS, ApJ . . 3.

[10] N. R. Tanvir, A. J. Levan, A. S. Fruchter, J. Hjorth, R. A. Hounsell, K. Wiersema et al., A $\hat{a} \breve{A} \ddot{Y}$ kilonovaâĂŹ associated with the short-duration İs-ray burst GRB 130603b, Nature 500 (2013) 547.

[11] Z.-P. Jin, K. Hotokezaka, X. Li, M. Tanaka, P. DâĂŹAvanzo, Y.-Z. Fan et al., The Macronova in GRB 050709 and the GRB-macronova connection, Nature Communications 7 (2016) 12898.

[12] D. A. Perley, B. D. Metzger, J. Granot, N. R. Butler, T. Sakamoto, E. Ramirez-Ruiz et al., GRB 080503: Implications of a Naked Short Gamma-Ray Burst Dominated by Extended Emission, The Astrophysical Journal 696 (2009) 1871. 
[13] H. Gao, B. Zhang, H.-J. LÃij and Y. Li, Searching for Magnetar-powered Merger-novae from Short GRBS, The Astrophysical Journal 837 (2017) 50.

[14] Z.-P. Jin, X. Li, H. Wang, Y.-Z. Wang, H.-N. He, Q. Yuan et al., Short GRBs: Opening Angles, Local Neutron Star Merger Rate, and Off-axis Events for GRB/GW Association, The Astrophysical Journal 857 (2018) 128.

[15] M. M. Kasliwal, O. Korobkin, R. M. Lau, R. Wollaeger and C. L. Fryer, Infrared Emission from Kilonovae: The Case of the Nearby Short Hard Burst GRB 160821b, The Astrophysical Journal Letters 843 (2017) L34.

[16] W. Fong, R. Margutti, R. Chornock, E. Berger, B. J. Shappee, A. J. Levan et al., The Afterglow and Early-type Host Galaxy of the Short GRB 150101b at $z=0.1343$, The Astrophysical Journal $\mathbf{8 3 3}$ (2016) 151.

[17] A. Goldstein, P. Veres, E. Burns, M. S. Briggs, R. Hamburg, D. Kocevski et al., An Ordinary Short Gamma-Ray Burst with Extraordinary Implications: Fermi -GBM Detection of GRB 170817a, The Astrophysical Journal Letters 848 (2017) L14.

[18] V. Savchenko, C. Ferrigno, E. Kuulkers, A. Bazzano, E. Bozzo, S. Brandt et al., INTEGRAL Detection of the First Prompt Gamma-Ray Signal Coincident with the Gravitational-wave Event GW170817, The Astrophysical Journal Letters 848 (2017) L15.

[19] D. A. Coulter, R. J. Foley, C. D. Kilpatrick, M. R. Drout, A. L. Piro, B. J. Shappee et al., Swope Supernova Survey 2017 a (SSS17a), the optical counterpart to a gravitational wave source, Science 358 (2017) 1556.

[20] E. Pian, P. D’Avanzo, S. Benetti, M. Branchesi, E. Brocato, S. Campana et al., Spectroscopic identification of r-process nucleosynthesis in a double neutron-star merger, Nature 551 (2017) 67.

[21] S. J. Smartt, T.-W. Chen, A. Jerkstrand, M. Coughlin, E. Kankare, S. A. Sim et al., A kilonova as the electromagnetic counterpart to a gravitational-wave source, Nature (2017) .

[22] M. Nicholl, E. Berger, D. Kasen, B. D. Metzger, J. Elias, C. BriceÃśo et al., The Electromagnetic Counterpart of the Binary Neutron Star Merger LIGO/Virgo GW170817. III. Optical and UV Spectra of a Blue Kilonova from Fast Polar Ejecta, The Astrophysical Journal 848 (2017) L18.

[23] N. R. Tanvir, A. J. Levan, C. GonzÃąlez-FernÃąndez, O. Korobkin, I. Mandel, S. Rosswog et al., The Emergence of a Lanthanide-rich Kilonova Following the Merger of Two Neutron Stars, The Astrophysical Journal 848 (2017) L27.

[24] L.-X. Li and B. Paczy ̊̊Ďski, Transient Events from Neutron Star Mergers, The Astrophysical Journal Letters 507 (1998) L59.

[25] B. D. Metzger, G. MartÃønez-Pinedo, S. Darbha, E. Quataert, A. Arcones, D. Kasen et al., Electromagnetic counterparts of compact object mergers powered by the radioactive decay of r-process nuclei, Monthly Notices of the Royal Astronomical Society 406 (2010) 2650.

[26] R. FernÃąndez and B. D. Metzger, Electromagnetic Signatures of Neutron Star Mergers in the Advanced LIGO Era, Annual Review of Nuclear and Particle Science 66 (2016) 23.

[27] V. A. Villar, J. Guillochon, E. Berger, B. D. Metzger, P. S. Cowperthwaite, M. Nicholl et al., The Combined Ultraviolet, Optical, and Near-infrared Light Curves of the Kilonova Associated with the Binary Neutron Star Merger GW170817: Unified Data Set, Analytic Models, and Physical Implications, The Astrophysical Journal 851 (2017) L21. 
[28] D. Kasen, B. Metzger, J. Barnes, E. Quataert and E. Ramirez-Ruiz, Origin of the heavy elements in binary neutron-star mergers from a gravitational-wave event, Nature 551 (2017) 80.

[29] A. Perego, D. Radice and S. Bernuzzi, AT 2017gfo: An Anisotropic and Three-component Kilonova Counterpart of GW170817, The Astrophysical Journal Letters 850 (2017) L37.

[30] D. Kasen, R. FernÃąndez and B. D. Metzger, Kilonova light curves from the disc wind outflows of compact object mergers, Monthly Notices of the Royal Astronomical Society 450 (2015) 1777.

[31] D. Radice, A. Perego, K. Hotokezaka, S. A. Fromm, S. Bernuzzi and L. F. Roberts, Binary Neutron Star Mergers: Mass Ejection, Electromagnetic Counterparts and Nucleosynthesis, arXiv:1809.11161 [astro-ph] (2018).

[32] S. Rosswog, J. Sollerman, U. Feindt, A. Goobar, O. Korobkin, R. Wollaeger et al., The first direct double neutron star merger detection: Implications for cosmic nucleosynthesis, Astronomy \& Astrophysics 615 (2018) A132.

[33] B. P. Gompertz, A. J. Levan, N. R. Tanvir, J. Hjorth, S. Covino, P. A. Evans et al., The Diversity of Kilonova Emission in Short Gamma-Ray Bursts, The Astrophysical Journal 860 (2018) 62.

[34] S. Ascenzi, M. W. Coughlin, T. Dietrich, R. J. Foley, E. Ramirez-Ruiz, S. Piranomonte et al., A luminosity distribution for kilonovae based on short gamma-ray burst afterglows, arXiv:1811.05506 [astro-ph, physics:gr-qc] (2018).

[35] B. P. Abbott, R. Abbott, T. D. Abbott, M. R. Abernathy, F. Acernese, K. Ackley et al., Prospects for observing and localizing gravitational-wave transients with Advanced LIGO, Advanced Virgo and KAGRA, Living Reviews in Relativity 21 (2018) 3.

[36] M. Punturo, M. Abernathy, F. Acernese, B. Allen, N. Andersson, K. Arun et al., The Einstein Telescope: a third-generation gravitational wave observatory, Classical and Quantum Gravity 27 (2010) 194002.

\section{DISCUSSION}

J. H. Beall: You show both a jet and a shell interacting with the external ISM. Is there a particular reason you did not just use a jet in your model?

G. Stratta: From two merging neutron stars we expect two main electromagnetic counterparts: 1) a non-thermal emission in the form of a collimated gamma-ray burst and afterglow produced by the interaction of a relativistic jet with the ISM 2) an isotropic, thermal emission (kilonova) generated by the radioactive decay of freshly synthesized heavy elements. The most recent theoretical interpretation of GW170817/short GRB 170817 and its afterglow invokes a structured jet model formed by a narrow, relativistic jet of half-opening angle 4.5 deg and a mildy relativistic broader jet ("cocoon", Mooley et al. 2018). The kilonova emission, contrary to the GRB counterpart, is expected to irradiate almost isotropically. To explain the observed properties of the kilonova AT2017gfo associated with GW170817 the existence of two main regions of emission has been invoked: a "blue" one that is confined near the polar regions, with an half-jet opening angle of $\sim 60 \mathrm{deg}$ (so not properly a jet) and a red one confined on the equatorial plane. The latter is thought to be the region where the most heavy elements are produced (e.g. lanthanides) that have also higher opacity (this is why optical emission is degraded towards the NIR "red" band). 